\title{
PASSIVITY AND PASSIVATION OF INTERCONNECTED TIME-DELAY MODELS OF REHEAT POWER SYSTEMS
}

\author{
MAGDI S. MAHMOUD AND ABDULLA ISMAIL
}

Received 10 March 2005; Accepted 24 January 2006

This paper investigates the problems of delay-dependent passivity and passivation of a class of linear interconnected time-delay systems with particular emphasis on multiarea reheat power systems. This class contains state delay in the dynamics and observation at the subsystem (local) level. A new state transformation is developed to exhibit the delay dependence in the system dynamics and a less conservative passivity-bounding inequality is incorporated. Through the analytical development, it is established that the passivity condition can be cast in a linear matrix inequality (LMI) format at the subsystem level thereby facilitating decentralized passivity analysis. For state-feedback passivation, it is proven that it is indifferent to use instantaneous or delayed decentralized state feedback. The case of dynamic output-feedback passivation is also treated. The analytical developments are simulated to a typical multiarea power system and the ensuing results show satisfactory performance.

Copyright (C 2006 M. S. Mahmoud and A. Ismail. This is an open access article distributed under the Creative Commons Attribution License, which permits unrestricted use, distribution, and reproduction in any medium, provided the original work is properly cited.

\section{Introduction}

Dynamical systems are being connected to form larger systems to meet the pressing demands and therefore problems of decentralized control and stabilization of interconnected systems are receiving considerable interests [1, 3-5, 11, 16, 21, 24, 26] where most of the effort focused on dealing with the interaction patterns. When the interconnected system involves delays [19], only few studies are available. In [12, 13], the focus has been on delays in the interaction patterns with the subsystem dynamics being known completely. There are various sources for delays including finite capabilities of information processing among different parts of the system, inherent phenomena like mass transport flow and recycling and/or byproduct of computational delays [19]. In [15], a class of uncertain systems is considered where the delays occur within the subsystems. Among 
the relevant topics is the passivity analysis and synthesis for classes of time-delay systems $[18,22,23,25]$ using classical definitions of passivity and positive realness $[2,7,27]$. These results show that passivity-based methods are highly effective and producing robust controllers to classes of time-delay with parametric uncertainties. In particular, when the time-delay factor is known, it is emphasized that delay-dependent passivity yields less conservative performance results. An alternative approach to interconnected power systems has been considered in [28] by representing the power system as Hamiltonian control system with dissipation and then using decentralized saturated nonlinear control to enhance the transient stability.

This paper has two major goals. First, it develops delay-dependent passivity and passivation criteria for a class of linear interconnected time-delay systems and designs decentralized state- and output-feedback controllers. Second, it applies these controllers to a class of interconnected reheat power systems. To achieve the first goal, we consider a less restrictive definition of passivity in dynamical systems [14] and employ a new state transformation to exhibit the delay-dependent system dynamics. Then we proceed to establish that the resulting passivity condition can be cast in a linear matrix inequality (LMI) format which can be conveniently solved by semidefinite programming techniques [7]. Following this, the problem of feedback passivation is examined and it is proven that when the state variables are available for measurements, it is indifferent to use instantaneous or delayed state feedback. This represents a pleasing avenue which enhances the effectiveness of the developed state transformation. Finally, the case of dynamic output-feedback passivation is treated and it is shown that the passivity conditions are cast in the form of system of equations and LMIs.

To achieve the second goal, we consider a class of interconnected reheat power systems, the model of the individual system possesses intrinsic time-delay features due to the feedback channel of reheat power as well as the finite processing capabilities in the governor and power system stabilizer. As a typical application, we consider a three-area reheat power system and use Matlab simulation to explore the effectiveness of the developed control strategy vis-à-vis standard control design approaches. The ensuing simulation results show the superiority of the analytical technique developed in this paper for a wide range of power operating conditions.

Looked at in this light, the developed results in this paper for delay-dependent passivity (Theorem 2.3), state-feedback passivation (Theorem 3.1), and output-feedback passivation (Theorem 4.1) give less conservative results than those of $[9,15-19,21-25]$ since they provide a new state transformation for delay-dependent passive analysis and synthesis. Additionally, our control approach is technically superior to the techniques of $[24,29]$ since it establishes delay-dependent dynamic controllers for interconnected time-delay systems.

Notations and facts. In the sequel, we use $W^{t}, W^{-1}$, and $\lambda(W)$ to denote, respectively, the transpose, the inverse, and the eigenvalues of the induced norm of any square matrix $W$. We use $W>0(<0)$ to denote a symmetric positive definite (negative definite). Sometimes, the arguments of a function will be omitted in the analysis when no confusion can arise. The symbol $\bullet$ will be used in some matrix expressions to induce a symmetric 
structure, that is, if given matrices $L=L^{t}$ and $R=R^{t}$ of appropriate dimensions, then

$$
\left[\begin{array}{cc}
L+M+\bullet & \bullet \\
N & R
\end{array}\right]=\left[\begin{array}{cc}
L+M+M^{t} & N^{t} \\
N & R
\end{array}\right] .
$$

Fact 1.1. For any real matrices $\Sigma_{1}$ and $\Sigma_{2}$ with appropriate dimensions, it follows for all $\alpha>0$ that

$$
\Sigma_{1} \Sigma_{2}+\Sigma_{2}^{t} \Sigma_{1}^{t} \leq \alpha^{-1} \Sigma_{1} \Sigma_{1}^{t}+\alpha \Sigma_{2}^{t} \Sigma_{2}
$$

\section{Passivity of interconnected time-delay systems}

We consider a class of interconnected time-delay systems $\Sigma_{o}$,

$$
\begin{gathered}
\Sigma_{o}: \dot{x}(t)=\left[A_{o}+E\right] x(t)+A_{d} x(t-\tau)+\Gamma w(t), \\
x(s)=\kappa(s), \quad s \in[-\tau, 0], \\
z(t)=G_{o} x_{j}(t)+G_{d} x_{j}(t-\tau)+\Phi w(t),
\end{gathered}
$$

to be composed of $n_{s}$ coupled subsystems $\Sigma_{o j}$ described by

$$
\begin{gathered}
\Sigma_{o j}: \dot{x}_{j}(t)=A_{o j} x_{j}(t)+A_{d j} x_{j}\left(t-\tau_{j}\right)+\sum_{k=1}^{n_{s}} E_{j k} x_{k}(t)+\Gamma_{j} w_{j}(t), \\
x_{j}(s)=\kappa_{j}(s), \quad s \in\left[-\tau_{j}, 0\right] \\
z_{j}(t)=G_{o j} x_{j}(t)+G_{d j} x_{j}\left(t-\tau_{j}\right)+\Phi_{j} w_{j}(t),
\end{gathered}
$$

where $x_{j}(t) \in \mathbb{R}^{n_{j}}$ is the state vector, $w_{j}(t) \in \mathbb{R}^{q_{j}}$ is the exogenous input (either a control input or reference signal), $z_{j}(t) \in \mathbb{R}^{q_{j}}$ is the controlled output, and $\tau_{j} \in\left[0, \tau_{j}^{*}\right]$ is a known constant delay factor. The matrices $A_{o j}, A_{d j}, G_{o j}, G_{d j}, \Gamma_{j}$, and $\Phi_{j}$ are known real constant matrices of appropriate dimensions. The links between the matrices of system $\Sigma_{o}$ and subsystems $\Sigma_{o j}, j=1, \ldots, n_{s}$, are quite standard. From now onwards, we focus on analysis and design at the subsystem level. Let $X_{j}\left(t, \kappa_{j}\right)$ denote the state trajectory of system $\Sigma_{o j}$ from the initial condition $\left\{x_{o j}, \kappa_{j}\right\}$ and let $X_{j}(t, 0)$ be the corresponding trajectory with zero initial conditions.

Definition 2.1. System $\Sigma_{o}$ is called passive if there exist scalars $\beta_{j} \geq 0, j=1, \ldots, n_{s}$, such that for all $t_{p j} \geq 0$ and for all $X_{j}(t, 0)$ of subsystems $\Sigma_{o j}, j=1, \ldots, n_{s}$,

$$
2 \int_{0}^{t_{p j}} w_{j}^{t}(s) z_{j}(s) d s \geq-\beta_{j} \int_{0}^{t_{p j}} w_{j}^{t}(s) w_{j}(s) d s .
$$




\section{Passivity and passivation}

Remark 2.2. It should be observed that Definition 2.1 provides less conservative performance results for a wide class of dynamical systems [14]. There are other alternative approaches based on port-controlled Hamiltonian systems with dissipation $[8,28]$ by considering the Hamiltonian function as the total energy (potential and kinetic energy) in the mechanical systems. These, however, require an additional a priori modeling effort in contrast to the direct state-space model (2.2)-(2.6) which permits incorporating timedelay factors.

Our purpose in this paper is to develop delay-dependent criteria for the interconnected time-delay system $\Sigma_{o}$ using local information from subsystems (2.5)-(2.6) that render the system internally stable and passive based on Definition 2.1 and subsequently construct methods for feedback passivation.

2.1. State transformation. Introduce the state transformation

$$
\sigma_{j}(t)=x_{j}(t)+\int_{t-\tau_{j}}^{t} A_{d j} x_{j}(s) d s
$$

into (2.5) to yield

$$
\begin{aligned}
\dot{\sigma}_{j}(t) & =\left[A_{o j}+A_{d j}\right] x_{j}(t)+\Gamma_{j} w_{j}(t)+\sum_{k=1}^{n_{s}} E_{j k} x_{k}(t) \\
& =A_{o d j} x(t)+\Gamma_{j} w_{j}(t)+\sum_{k=1}^{n_{s}} E_{j k} x_{k}(t) .
\end{aligned}
$$

Define the augmented state vector

$$
\zeta_{j}(t)=\left[\begin{array}{c}
\sigma_{j}(t) \\
\varepsilon_{j} x_{j}(t)
\end{array}\right] \in \mathbb{R}^{2 n} .
$$

By combining (2.5) and (2.8)-(2.10) and taking the limit $\varepsilon_{j} \rightarrow 0$, we obtain the transformed system

$$
\begin{gathered}
\left(\Sigma_{T_{o j}}\right): \dot{\zeta}_{j}(t)=\Lambda_{o j} \zeta_{j}(t)+\int_{t-\tau_{j}}^{t} \Upsilon_{j} \zeta_{j}(s) d s+\bar{\Gamma}_{j} w_{j}(t)+\sum_{k=1}^{n_{s}} \bar{E}_{j k} \zeta_{k}(t), \\
\zeta_{j}(t)=\bar{\kappa}_{j}(t), \quad t \in\left[-2 \tau_{j}, 0\right] \\
z_{j}(t)=\bar{G}_{j} \zeta_{j}(t)+\bar{G}_{d j} \zeta\left(t-\tau_{j}\right)+\Phi_{j} w_{j}(t),
\end{gathered}
$$


along with

$$
\begin{aligned}
\bar{\Gamma}_{j} & =\left[\begin{array}{c}
\Gamma_{j} \\
0
\end{array}\right], \quad \bar{G}_{o j}=\left[\begin{array}{ll}
0 & G_{o j}
\end{array}\right], \quad \bar{G}_{d j}=\left[\begin{array}{ll}
0 & G_{d j}
\end{array}\right], \quad \bar{E}_{j k}=\left[\begin{array}{cc}
0 & E_{j k} \\
0 & 0
\end{array}\right], \\
\Lambda_{o j} & =\left[\begin{array}{cc}
0 & A_{o d j} \\
-I & I
\end{array}\right], \quad \Upsilon_{j}=\left[\begin{array}{cc}
0 & 0 \\
0 & A_{d j}
\end{array}\right], \quad R_{j}=\left[\begin{array}{cc}
R_{\sigma j} & 0 \\
0 & R_{x j}
\end{array}\right], \\
E_{1} & \left.=\left[\begin{array}{l}
I \\
0
\end{array}\right], \quad E_{2}=\left[\begin{array}{l}
0 \\
I
\end{array}\right], \quad \begin{array}{l}
\bar{P}_{j}=U_{j} \mathbb{P}_{j}, \quad U_{j}=\left[\begin{array}{cc}
I_{j} & 0 \\
0 & 0
\end{array}\right], \quad \mathbb{P}_{j}=\left[\begin{array}{cc}
P_{\sigma j} & 0 \\
P_{d j} & P_{x j}
\end{array}\right], \\
(2.14) \\
\Pi_{o j}
\end{array}\right]\left[\begin{array}{c}
-P_{x j}+P_{d j}^{t} \\
+P_{\sigma j}^{t} A_{o d j}+ \\
P_{\sigma j}^{t} \sum_{k=1}^{n_{s}} E_{k j} \\
P_{x j}+P_{x j}^{t}+ \\
R_{x j}+\tau_{j} Q_{j}
\end{array}\right], \quad R_{\sigma j}=\left[\begin{array}{c}
\tau_{j} P_{d j}^{t} A_{d j} \\
\tau_{j} P_{x j}^{t} A_{d j}
\end{array}\right], \quad \Pi_{t j}=\left[\begin{array}{c}
P_{\sigma j}^{t} \Gamma_{j} \\
-G_{o j}^{t}
\end{array}\right] .
\end{aligned}
$$

Theorem 2.3. Consider system $\Sigma_{T_{o j}}$. Given matrices $0<Q_{j}=Q_{j}^{t} \in \mathbb{R}^{n_{j} \times n_{j}}, 0<R_{j}=R_{j}^{t} \in$ $\mathbb{R}^{2 n_{j} \times 2 n_{j}}$, let matrices $0<P_{\sigma j}, P_{d j}, P_{x j}$ and scalar $\beta_{j} \geq 0$ satisfy the linear matrix inequalities (LMIs)

$$
\left[\begin{array}{ccc}
\Pi_{o j} & \Pi_{r j} & \Pi_{t j} \\
\bullet & -\tau_{j} Q_{j} & 0 \\
\bullet & \bullet & -\beta_{j} I-\Phi_{j}^{t} \\
\bullet & & -\Phi_{j}+G_{d j} R_{x j}^{-1} G_{d j}^{t}
\end{array}\right]<0, \quad\left[\begin{array}{ccc}
-\Phi_{j}-\Phi_{j}^{t} & \beta_{j} I & G_{d j} \\
\bullet & \beta_{j} I & 0 \\
\bullet & \bullet & -R_{x j}
\end{array}\right]<0,
$$

then system $\Sigma_{T_{o j}}$ is passive in the sense of Definition 2.1 .

Proof. For given $Q_{j}=Q_{j}^{t}>0$ and $R_{j}=R_{j}^{t}>0$, let the Lyapunov functional $V_{j}(\cdot)$ of the transformed system (2.11)-(2.13) be selected as

$$
V_{j}\left(t, \zeta_{j}\right)=\zeta_{j}^{t}(t) \bar{P}_{j}^{t} \zeta_{j}(t)+\int_{t-\tau_{j}}^{t} \zeta_{j}^{t}(s) R_{j} \zeta_{j}(s) d s+\int_{t-\tau_{j}}^{t} \int_{t+\theta_{j}}^{t} \zeta_{j}^{t}(s) E_{2} Q_{j} E_{2}^{t} \zeta_{j}(s) d s d \theta_{j}
$$

Evaluating of the derivative of $V\left(t, \zeta_{j}\right)$ with respect to $t$ yields

$$
\begin{aligned}
\dot{V}_{j}\left(t, \zeta_{j}\right)= & 2 \zeta_{j}^{t}(t) \bar{P}_{j}^{t} \dot{\zeta}_{j}(t)+\tau_{j} \zeta_{j}^{t}(t) E_{2} Q_{j} E_{2}^{t} \zeta_{j}(t)-\int_{-\tau_{j}}^{0} \zeta_{j}^{t}\left(\theta_{j}\right) E_{2} Q_{j} E_{2}^{t} \zeta_{j}\left(\theta_{j}\right) d \theta_{j} \\
& +\zeta_{j}^{t}(t) R_{j} \zeta_{j}(t)-\zeta_{j}^{t}\left(t-\tau_{j}\right) R_{j} \zeta_{j}\left(t-\tau_{j}\right) .
\end{aligned}
$$




\section{Passivity and passivation}

Focusing on the first term of (2.18) and using (2.13)-(2.17), we obtain

$$
\begin{aligned}
2 \zeta_{j}^{t}(t) \bar{P}_{j}^{t} \dot{\zeta}_{j}(t)= & 2 \sigma^{t}(t) P_{\sigma}^{t} \dot{\sigma}(t) \\
= & 2 \zeta_{j}^{t}(t) \mathbb{P}_{j}^{t}\left[\begin{array}{c}
\dot{\sigma}_{j}(t) \\
0
\end{array}\right]=2 \zeta_{j}^{t}(t) \mathbb{P}_{j}^{t}\left[\begin{array}{c}
A_{o d j} x(t)+\Gamma_{j} w_{j}(t)+\sum_{k=1}^{n_{s}} E_{j k} x_{k}(t) \\
-\sigma_{j}(t)+x_{j}(t)+\int_{t-\tau_{j}}^{t} A_{d j} x(s) d s
\end{array}\right] \\
= & 2 \zeta_{j}^{t}(t) \mathbb{P}_{j}^{t} \Lambda_{o j} \zeta_{j}(t)+2 \zeta_{j}^{t}(t) \mathbb{P}_{j}^{t} \bar{\Gamma}_{j} w_{j}(t)+2 \int_{t-\tau_{j}}^{t} \zeta_{j}^{t}(s) \mathbb{P}_{j}^{t} \Upsilon_{j} \zeta_{j}(s) d s \\
& +2 \zeta_{j}^{t}(t) \mathbb{P}_{j}^{t} \sum_{k=1}^{n_{s}} \bar{E}_{j k} \zeta_{k}(t) .
\end{aligned}
$$

Hence, it follows from (2.18)-(2.19) that

$$
\begin{aligned}
\dot{V}_{j}\left(t, \zeta_{j}\right)= & \zeta_{j}^{t}(t)\left[\Lambda_{o j}^{t} \mathbb{P}_{j}+\mathbb{P}_{j}^{t} \Lambda_{o j}+R_{j}\right] \zeta_{j}(t)+2 \zeta_{j}^{t}(t) \mathbb{P}_{j}^{t} \bar{\Gamma}_{j} w_{j}(t) \\
& +2 \int_{t-\tau_{j}}^{t} \zeta_{j}^{t}(s) \mathbb{P}_{j}^{t} \Upsilon_{j} \zeta_{j}(s) d s+\tau_{j} \zeta_{j}^{t}(t) E_{2} Q_{j} E_{2}^{t} \zeta_{j}(t)-\zeta^{t}\left(t-\tau_{j}\right) R_{j} \zeta_{j}\left(t-\tau_{j}\right) \\
& -\int_{t-\tau_{j}}^{t} \zeta_{j}^{t}(s) E_{2} Q_{j} E_{2}^{t} \zeta_{j}(s) d s+2 \zeta_{j}^{t}(t) \mathbb{P}_{j}^{t} \sum_{k=1}^{n_{s}} \bar{E}_{j k} \zeta_{k}(t) .
\end{aligned}
$$

By Fact 1.1, we have

$$
\begin{aligned}
2 \int_{t-\tau_{j}}^{t} \zeta_{j}^{t}(s) \mathbb{P}_{j}^{t} \Upsilon_{j} \zeta_{j}(s) d s & =2 \int_{t-\tau_{j}}^{t} \zeta_{j}^{t}(s) \mathbb{P}_{j}^{t} E_{2} A_{d j} x_{j}(s) d s \\
& \leq \tau_{j} \zeta_{j}^{t}(t) \mathbb{P}_{j}^{t} E_{2} A_{d j} Q_{j}^{-1} A_{d j}^{t} E_{2}^{t} \mathbb{P}_{j}^{t} \zeta_{j}(t)+\int_{t-\tau_{j}}^{t} x_{j}^{t}(s) Q_{j} x_{j}(s) d s \\
& =\tau_{j} \zeta_{j}^{t}(t) \mathbb{P}_{j}^{t} E_{2} A_{d j} Q_{j}^{-1} A_{d j}^{t} E_{2}^{t} \mathbb{P}_{j} \zeta_{j}(t)+\int_{t-\tau_{j}}^{t} \zeta_{j}^{t}(s) E_{2} Q_{j} E_{2}^{t} \zeta_{j}(s) d s .
\end{aligned}
$$

From (2.20)-(2.21), we get

$$
\begin{aligned}
\dot{V}_{j}\left(t, \zeta_{j}\right)=\zeta_{j}^{t}(t)[ & \Lambda_{o j}^{t} \mathbb{P}_{j}+\mathbb{P}_{j}^{t} \Lambda_{o j}+R_{j}+\tau_{j} \mathbb{P}^{t} E_{2} A_{d j} Q_{j}^{-1} A_{d j}^{t} E_{2}^{t} \mathbb{P}_{j}+\tau_{j} E_{2} Q_{j} E_{2}^{t} \\
& \left.+\mathbb{P}_{j}^{t} \sum_{k=1}^{n_{s}} \bar{E}_{j k}+\sum_{k=1}^{n_{s}} \bar{E}_{j k}^{t} \mathbb{P}_{j}\right] \zeta_{j}(t)+2 \zeta_{j}^{t}(t) \mathbb{P}_{j}^{t} \bar{\Gamma}_{j} w_{j}(t)-\zeta_{j}^{t}\left(t-\tau_{j}\right) R_{j} \zeta_{j}\left(t-\tau_{j}\right) .
\end{aligned}
$$


By incorporating the identity $\sum_{j=1}^{n_{s}} \sum_{k=1}^{n_{s}} \bar{E}_{j k} \zeta_{k}(t)=\sum_{k=1}^{n_{s}} \sum_{j=1}^{n_{s}} \bar{E}_{k j} \zeta_{j}(t)$ and (2.13) into (2.22) and completing the squares, it yields

$$
\begin{aligned}
& \dot{V}_{j}\left(t, \zeta_{j}\right)-2 z_{j}^{t}(t) w_{j}(t)-\beta_{j} w_{j}^{t}(t) w_{j}(t) \\
& =\zeta_{j}^{t}(t)\left[\Lambda_{o j}^{t} \mathbb{P}_{j}+\mathbb{P}_{j}^{t} \Lambda_{o j}+R_{j}+\tau_{j} \mathbb{P}^{t} E_{2} A_{d j} Q_{j}^{-1} A_{d j}^{t} E_{2}^{t} \mathbb{P}_{j}\right. \\
& \left.\quad+\tau_{j} E_{2} Q_{j} E_{2}^{t}+\mathbb{P}_{j}^{t} \sum_{k=1}^{n_{s}} \bar{E}_{j k}+\sum_{k=1}^{n_{s}} \bar{E}_{j k}^{t} \mathbb{P}_{j}\right] \zeta_{j}(t)+2 \zeta_{j}^{t}(t) \mathbb{P}_{j}^{t} \bar{\Gamma}_{j} w_{j}(t) \\
& -\beta_{j} w_{j}^{t}(t) w_{j}(t)-\zeta_{j}^{t}(t) \bar{G}_{j}^{t} w_{j}(t)-w_{j}^{t}(t) \bar{G}_{j} \zeta_{j}(t)-w_{j}^{t}(t)\left(\Phi_{j}+\Phi_{j}^{t}\right) w_{j}(t) \\
& -\zeta_{j}^{t}\left(t-\tau_{j}\right) R_{j} \zeta_{j}\left(t-\tau_{j}\right)-\zeta_{j}^{t}\left(t-\tau_{j}\right) \bar{G}_{d j}^{t} w_{j}(t)-w_{j}^{t}(t) \bar{G}_{d j} \zeta_{j}\left(t-\tau_{j}\right) \\
& <\zeta_{j}^{t}(t)\left[\Lambda_{o j}^{t} \mathbb{P}_{j}+\mathbb{P}_{j}^{t} \Lambda_{o j}+R_{j}+\tau_{j} \mathbb{P}^{t} E_{2} A_{d j} Q_{j}^{-1} A_{d j}^{t} E_{2}^{t} \mathbb{P}_{j}+\mathbb{P}_{j}^{t} \sum_{k=1}^{n_{s}} \bar{E}_{k j}+\sum_{k=1}^{n_{s}} \bar{E}_{k j}^{t} \mathbb{P}_{j}\right. \\
& \left.\quad+\tau_{j} E_{2} Q_{j} E_{2}^{t}+\left(\mathbb{P}_{j}^{t} \bar{\Gamma}_{j}-\bar{G}_{j}^{t}\right)\left(\Phi_{j}^{t}+\Phi_{j}+\beta_{j} I-\bar{G}_{d j} R_{j}^{-1} \bar{G}_{d j}^{t}\right){ }^{-1}\left(\bar{\Gamma}_{j}^{t} \mathbb{P}_{j}-\bar{G}_{j}\right)\right] \zeta_{j}(t) .
\end{aligned}
$$

Applying the Schur complements to (2.23) and in view of LMIs (2.15) with (2.16), it follows by integrating (2.23) with respect to $t$ over the period $0 \mapsto t_{p}$ that (2.7) holds and hence system $\Sigma_{T_{o}}$ is passive in the sense of Definition 2.1.

\section{State-feedback passivation}

We now build on the foregoing results by considering the feedback passivation problem, that is, designing a feedback controller to render the closed-loop time-delay system passive. Extending on system $\left(\Sigma_{J o}\right)$, we consider a class of time-delay systems of the form

$$
\begin{gathered}
\Sigma_{j}: \dot{x}_{j}(t)=A_{o j} x_{j}(t)+A_{d j} x_{j}\left(t-\tau_{j}\right)+\sum_{k=1}^{n_{s}} E_{j k} x_{k}(t)+B_{o j} u_{j}(t)+\Gamma_{j} w_{j}(t), \\
x_{j}(s)=\kappa_{j}(s), \quad s \in\left[-\tau_{j}, 0\right] \\
z_{j}(t)=G_{o j} x_{j}(t)+G_{d j} x_{j}\left(t-\tau_{j}\right)+\Phi_{j} w_{j}(t),
\end{gathered}
$$

where $u_{j}(t) \in \mathbb{R}^{r_{j}}$ is the part of the control input used for feedback. Considering initially the case of instantaneous state-feedback passivation, we use the decentralized control law $u_{j}(t)=K_{j} x_{j}(t)$ such that its application to (3.2) under the transformation (2.8) yields

$$
\dot{\sigma}_{j}(t)=A_{o k j} x_{j}(t)+\sum_{k=1}^{n_{s}} E_{j k} x_{k}(t)+\Gamma_{j} w_{j}(t), \quad A_{o k j}=A_{o j}+B_{o j} K_{j} .
$$


8 Passivity and passivation

Then the transformed system becomes

$$
\begin{gathered}
\left(\Sigma_{T k}\right): \dot{\zeta}_{j}(t)=\Lambda_{o k j} \zeta_{j}(t)+\int_{t-\tau_{j}}^{t} \Upsilon_{j} \zeta_{j}(s) d s+\sum_{k=1}^{n_{s}} \bar{E}_{j k} \zeta_{k}(t)+\bar{\Gamma}_{j} w_{j}(t), \\
\zeta_{j}(s)=\bar{\kappa}_{j}(s), \quad s \in\left[-2 \tau_{j}, 0\right] \\
z_{j}(t)=\bar{G}_{o j} \zeta_{j}(t)+\bar{G}_{d j} \zeta_{j}\left(t-\tau_{j}\right)+\Phi_{j} w_{j}(t),
\end{gathered}
$$

where

$$
\Lambda_{o k j}=\left[\begin{array}{cc}
0 & A_{o d j}+B_{o j} K_{j} \\
-I & I
\end{array}\right]
$$

Define the following matrices:

$$
\begin{gathered}
\bar{A}_{\text {odj }}^{t}=\left[\begin{array}{ll}
A_{o d j}^{t} & I
\end{array}\right], \quad Z_{j}=\left[\begin{array}{c}
0 \\
X_{\sigma j}
\end{array}\right], \quad \bar{B}_{o j}=\left[\begin{array}{c}
B_{o j} \\
0
\end{array}\right], \\
Y_{j}=\left[\begin{array}{ll}
X_{d j} & X_{x j}
\end{array}\right], \quad W_{j}=\left[\begin{array}{ll}
W_{d j} & W_{x j}
\end{array}\right],
\end{gathered}
$$

where

$$
\mathbb{P}_{j}^{-1}=\left[\begin{array}{cc}
X_{\sigma j} & 0 \\
X_{d j} & X_{x j}
\end{array}\right], \quad X_{\sigma j}=P_{\sigma j}^{-1}, X_{x j}=P_{x j}^{-1}, X_{d j}=-X_{x j} P_{d j} X_{\sigma j} .
$$

The following theorem establishes the main result.

Theorem 3.1. Consider system $\Sigma_{T k}$. Given matrix $0<Q_{j}=Q_{j}^{t} \in \mathbb{R}^{n_{j} \times n_{j}}$, let matrices $Y_{j}$, $Z_{j}, W_{j}$ and scalar $\beta_{j} \geq 0$ satisfy the system of LMIs

$$
\left[\begin{array}{cccc}
\Pi_{c j} & \tau_{j} E_{2} A_{d j} & \tau_{j} Y_{j}^{t} E_{2} & \bar{\Gamma}_{j}-Y_{j}^{t} G_{o j}^{t} \\
\bullet & -\tau_{j} Q_{j} & 0 & 0 \\
\bullet & \bullet & -\tau_{j} Q_{j} & 0 \\
\bullet & \bullet & \bullet & -\beta_{j} I-\Phi_{j}^{t} \\
& & & -\Phi_{j}+\bar{G}_{d j} R_{j}^{-1} \bar{G}_{d j}^{t}
\end{array}\right]<0, \quad\left[\begin{array}{ccc}
-\Phi_{j}-\Phi_{j}^{t} & \beta_{j} I & \bar{G}_{d j} \\
\bullet & \beta_{j} I & 0 \\
\bullet & \bullet & -R_{j}
\end{array}\right]<0
$$

then system $\Sigma_{T k}$ is passive under the control law $u(t)=K x(t)$ in the sense of Definition 2.1, where

$$
\Pi_{c j}=Y_{j}^{t} \bar{A}_{o d j}^{t}+\bar{A}_{o d j} Y_{j}-E_{1} Z_{j}^{t}-Z_{j} E_{1}^{t}+\bar{B}_{o j} W_{j}+W_{j}^{t} \bar{B}_{o j}^{t}+\sum_{k=1}^{n_{s}} E_{k j} E_{1} Y_{j}+Y_{j}^{t} E_{1}^{t} \sum_{k=1}^{n_{s}} E_{k j}^{t}
$$

and the state-feedback gain is given by $K_{j}=\left[W_{j} E_{2}\right]\left[Y_{j} E_{2}\right]^{-1}$. 
Proof. By Theorem 2.3 and taking into consideration (2.14)-(2.15), it is easy to see that passivity of system $\Sigma_{T k}$ implies that the inequality

$$
\begin{aligned}
& \Lambda_{o j}^{t} \mathbb{P}_{j}+\mathbb{P}_{j}^{t} \Lambda_{o j}+R_{j}+\tau_{j} \mathbb{P}^{t} E_{2} A_{d j} Q_{j}^{-1} A_{d j}^{t} E_{2}^{t} \mathbb{P}_{j}+\mathbb{P}_{j}^{t} \sum_{k=1}^{n_{s}} \bar{E}_{k j}+\sum_{k=1}^{n_{s}} \bar{E}_{k j}^{t} \mathbb{P}_{j} \\
& \quad+\tau_{j} E_{2} Q_{j} E_{2}^{t}+\left(\mathbb{P}_{j}^{t} \bar{\Gamma}_{j}-\bar{G}_{j}^{t}\right)\left(\Phi_{j}^{t}+\Phi_{j}+\beta_{j} I-\bar{G}_{d j} R_{j}^{-1} \bar{G}_{d j}^{t}\right)^{-1}\left(\bar{\Gamma}_{j}^{t} \mathbb{P}_{j}-\bar{G}_{j}\right)<0
\end{aligned}
$$

holds. Premultiplying (3.11) by $\mathbb{P}_{j}^{-t}$, postmultiplying by $\mathbb{P}_{j}^{-1}$, using (3.7)-(3.8) and the Schur complements, we obtain the LMI (3.9) and the proof is completed.

Remark 3.2. Had we considered delayed state-feedback passivation using the decentralized control law $u_{j}(t)=K_{d j} x_{j}\left(t-\tau_{j}\right)$, we would have used the state transformation as

$$
\sigma_{j}(t)=x_{j}(t)+\int_{t-\tau_{j}}^{t}\left[A_{d j}+B_{o j} K_{d j}\right] x(s) d s
$$

On substituting (3.12) into (3.2) with (2.14)-(2.15), it yields

$$
\dot{\sigma}_{j}(t)=\left[A_{o d j}+B_{o j} K_{d j}\right] x_{j}(t)+\sum_{k=1}^{n_{s}} E_{j k} x_{k}(t)+\Gamma_{j} w_{j}(t) .
$$

A little algebra gives the transformed system

$$
\begin{gathered}
\left(\Sigma_{T d k}\right): \dot{\zeta}_{j}(t)=\Lambda_{o d k j} \zeta_{j}(t)+\int_{t-\tau_{j}}^{t} \Upsilon_{j} \zeta_{j}(s) d s+\sum_{k=1}^{n_{s}} \bar{E}_{j k} \zeta_{k}(t)+\bar{\Gamma}_{j} w_{j}(t), \\
\zeta_{j}(s)=\bar{\kappa}_{j}(s), \quad s \in\left[-2 \tau_{j}, 0\right] \\
z_{j}(t)=\bar{G}_{o j} \zeta_{j}+\bar{G}_{d j} \zeta_{j}\left(t-\tau_{j}\right)+\Phi_{j} w_{j}(t),
\end{gathered}
$$

where

$$
\Lambda_{o d k j}=\left[\begin{array}{cc}
0 & A_{o d j}+B_{o j} K_{d j} \\
-I & I
\end{array}\right]
$$

Taking into account the matrices of (3.7)-(3.8), we could have established a result parallel to that of Theorem 3.1 by using $K_{j} \rightarrow K_{d j}$. It is thus not surprising to find that results on both instantaneous and delayed decentralized state-feedback passivation are equivalent. This, in fact, strengthens the state transformations (2.8) or (3.12) as vehicles to derive pertinent delay-dependent dynamic models. 


\section{Output-feedback passivation}

We make another generalization of the previous section by considering passivation using dynamic output feedback for the system

$$
\begin{gathered}
\left(\Sigma_{J O}\right): \dot{x}_{j}(t)=A_{o j} x(t)+A_{d j} x_{j}\left(t-\tau_{j}\right)+B_{o j} u_{j}(t)+\sum_{k=1}^{n_{s}} E_{j k} x_{k}(t)+\Gamma_{j} w_{j}(t), \\
x_{j}(s)=\kappa_{j}(s) \quad s \in\left[-\tau_{j}, 0\right] \\
y_{j}(t)=C_{o j} x_{j}(t)+E_{o j} x_{j}\left(t-\tau_{j}\right)+D_{o j} u_{j}(t), \\
z_{j}(t)=G_{o j} x_{j}(t)+G_{d j} x_{j}\left(t-\tau_{j}\right)+\Phi_{j} w_{j}(t)
\end{gathered}
$$

where $y_{j}(t)$ is the vector of output measurements. We consider the following dynamic feedback controller:

$$
\begin{gathered}
\left(\Sigma_{C j}\right): \dot{x}_{c j}(t)=A_{o j} x_{c j}(t)+B_{o j} u_{j}(t)+K_{c j}\left[y_{j}(t)-C_{o j} x_{c j}(t)\right] \\
u_{j}(t)=G_{c j} x_{c j}(t)
\end{gathered}
$$

where $K_{c j}, G_{c j}$ are the unknown gains. From (4.2)-(4.5), we get the combined system

$$
\begin{gathered}
\left(\Sigma_{J C O j}\right): \dot{\chi}_{j}(t)=\widehat{A}_{o j} \chi_{j}(t)+\widehat{A}_{d j} \chi_{j}\left(t-\tau_{j}\right)+\sum_{k=1}^{n_{s}} \bar{E}_{j k} \chi_{k}(t)+\bar{\Gamma}_{j} w_{j}(t), \\
z_{j}(t)=\hat{G}_{o j} \chi_{j}(t)+\widehat{G}_{d j} \chi_{j}\left(t-\tau_{j}\right)+\Phi_{j} w_{j}(t),
\end{gathered}
$$

where

$$
\begin{gathered}
\chi_{j}(t)=\left[\begin{array}{c}
x_{j}(t) \\
x_{c j}(t)
\end{array}\right], \quad \hat{A}_{o j}=\left[\begin{array}{cc}
A_{o j} & B_{o j} G_{c j} \\
K_{c j} C_{o j} & A_{c}+B_{o} G_{c}+K_{c}\left(D_{o} G_{c}-C_{o}\right)
\end{array}\right], \\
\hat{A}_{d j}=\left[\begin{array}{cc}
A_{d j} & 0 \\
K_{c j} E_{o j} & 0
\end{array}\right], \quad \hat{G}_{o j}=\left[\begin{array}{ll}
G_{o j} & 0
\end{array}\right], \quad \hat{G}_{d j}=\left[\begin{array}{ll}
G_{d j} & 0
\end{array}\right] .
\end{gathered}
$$

Introducing the state transformation

$$
\hat{\sigma}_{j}(t)=\chi_{j}(t)+\int_{t-\tau_{j}}^{t} \widehat{A}_{d j} x(s) d s
$$

which converts (4.6) into

$$
\begin{aligned}
\dot{\hat{\sigma}}_{j}(t) & =\left[\widehat{A}_{o j}+\widehat{A}_{d j}\right] \chi_{j}(t)+\sum_{k=1}^{n_{s}} \bar{E}_{j k} \chi_{k}(t)+\widehat{\Gamma}_{j} w_{j}(t) \\
& =\hat{A}_{o d j} \chi_{j}(t)+\sum_{k=1}^{n_{s}} \bar{E}_{j k} \chi_{k}(t)+\widehat{\Gamma}_{j} w_{j}(t),
\end{aligned}
$$


the transformed system becomes

$$
\begin{gathered}
\left(\Sigma_{T_{C O j}}\right): \dot{\xi}_{j}(t)=\left[\begin{array}{c}
\hat{\sigma}_{j} \\
\dot{\chi}_{j}
\end{array}\right]=\Lambda_{c o j} \xi_{j}(t)+\int_{t-\tau_{j}}^{t} \Upsilon_{c o j} \xi_{j}(s) d s+\sum_{k=1}^{n_{s}} \widehat{E}_{j k} \xi_{k}(t)+\widehat{\Gamma}_{j} w_{j}(t), \\
z_{j}(t)=G_{c o j} \xi_{j}(t)+G_{d o j} \xi_{j}\left(t-\tau_{j}\right)+\Phi_{j} w_{j}(t),
\end{gathered}
$$

where

$$
\begin{gathered}
\hat{\Gamma}_{j}=\left[\begin{array}{c}
\bar{\Gamma}_{j} \\
0
\end{array}\right], \quad G_{c o j}=\left[\begin{array}{ll}
0 & \hat{G}_{o j}
\end{array}\right], \quad G_{d o j}=\left[\begin{array}{ll}
0 & \hat{G}_{d j}
\end{array}\right], \quad \Lambda_{c o j}=\left[\begin{array}{cc}
0 & \hat{A}_{o d j} \\
-I & I
\end{array}\right], \\
\Upsilon_{c o j}=\left[\begin{array}{cc}
0 & 0 \\
0 & \hat{A}_{d j}
\end{array}\right], \quad \hat{E}_{j k}=\left[\begin{array}{cc}
0 & \bar{E}_{j k} \\
0 & 0
\end{array}\right] .
\end{gathered}
$$

By using the Schur complements, it is not difficult to deduce from Theorem 2.3 that given matrix $\mathscr{Q}_{j}=2_{j}^{t} \in \mathbb{R}^{2 n_{j} \times 2 n_{j}}, \mathscr{R}_{j}=\mathscr{R}_{j}^{t} \in \mathbb{R}^{4 n_{j} \times 4 n_{j}}$, it follows that system $\left(\Sigma_{T_{\mathrm{CO} j}}\right)$ is passive if there exists matrix $\Omega_{j}=\Omega_{j}^{t} \in \mathbb{R}^{4 n_{j} \times 4 n_{j}}$ satisfying the inequality

$$
\begin{aligned}
& \Lambda_{c o j}^{t} \Omega_{j}+\Omega_{j}^{t} \Lambda_{c o j}+\tau_{j} E_{2} \hat{A}_{d j} \mathscr{Q}_{j} \hat{A}_{d_{j}}^{t} E_{2}^{t}+\tau_{j} \Omega_{j}^{t} E_{2} \mathscr{2}_{j} E_{2}^{t} \Omega_{j}+\mathscr{R}_{j}+\Omega_{j}^{t} \sum_{k=1}^{n_{s}} \hat{E}_{j k}+\sum_{k=1}^{n_{s}} \hat{E}_{j k}^{t} \Omega_{j} \\
& \quad+\left(\Omega_{j}^{t} \hat{\Gamma}_{j}-G_{c o j}^{t}\right)\left(\Phi_{j}^{t}+\Phi_{j}+\beta_{j} I-G_{d j} R_{j}^{-1} G_{d j}^{t}\right)^{-1}\left(\hat{\Gamma}_{j}^{t} \Omega_{j}-G_{c o j}\right)<0,
\end{aligned}
$$

where

$$
\begin{gathered}
\Omega_{j}=\left[\begin{array}{cc}
\Omega_{\sigma j} & 0 \\
\Omega_{d j} & \Omega_{x j}
\end{array}\right], \quad \mathscr{Q}_{j}=\left[\begin{array}{cc}
\mathscr{2}_{\sigma j} & 0 \\
0 & \mathscr{Q}_{x j}
\end{array}\right], \quad \mathscr{R}_{j}=\left[\begin{array}{cc}
R_{j} & 0 \\
0 & 0
\end{array}\right], \\
H_{j}=\Phi_{j}^{t}+\Phi_{j}+\beta_{j} I-G_{d j} R_{j}^{-1} G_{d j}^{t} .
\end{gathered}
$$

We are now in position to present the following result.

Theorem 4.1. System $\Sigma_{J C O}$ is passive by the dynamic output feedback controller $\Sigma_{C j}$ if, given matrices $0<\mathscr{2}_{\sigma j}=\mathscr{2}_{\sigma j}^{t}, 0<\mathscr{2}_{x j}=\mathscr{2}_{x j}^{t}, 0<\mathscr{R}_{\sigma 1 j}=\mathscr{R}_{\sigma 1 j}^{t}, 0<\mathscr{R}_{x 1 j}=\mathscr{R}_{x 1 j}^{t}, 0<\mathscr{R}_{x 2 j}=$ $\mathscr{R}_{x 2 j}^{t}$, there exist matrices $\Omega_{\sigma_{1 j}}, \Omega_{\sigma_{2 j}}, \Omega_{d_{1 j}}, \Omega_{d_{2 j}}, \Omega_{x_{1 j}}, \Omega_{x_{2 j}}$ and scalar $\beta_{j} \geq 0$ satisfying the 
system of equations and LMIs

$$
\begin{gathered}
{\left[\begin{array}{cc}
\Pi_{d 1 j} & \tau_{j} \Omega_{d_{1 j}}^{t} \\
\bullet & -\tau_{j} 2_{\sigma j}
\end{array}\right]<0,\left[\begin{array}{ccc}
\Omega_{x_{2 j}}+\Omega_{x_{2 j}}^{t}+\mathscr{R}_{x 2 j} & \tau \Omega_{x_{2 j}}^{t} & \tau_{j} L_{j} \\
\bullet & -\tau_{j} 2_{2 j} & 0 \\
\bullet & \bullet & -\tau_{j} 2_{x j}
\end{array}\right]<0,} \\
{\left[\begin{array}{cc}
\Pi_{d 2 j} & \tau \Omega_{d_{2 j}}^{t} \\
\bullet & -\tau_{j} 2_{x j}
\end{array}\right]<0,} \\
\Omega_{\sigma_{1 j}}=\Omega_{d_{1 j}}+\Omega_{x_{1 j}}^{t} A_{o d j}^{t}+\tau_{j} \Omega_{x_{1 j}}^{t} 2_{\sigma j} \Omega_{d_{1 j}}-\Gamma_{j} H_{j} G_{o j} \Omega_{x_{1 j}}^{t}, \\
\bullet \\
\bullet \\
\Omega_{\sigma_{2 j}}=\Omega_{d_{2 j}}+\Omega_{x_{2 j}}^{t}\left(A_{o j}+B_{o j} D_{o j}^{t} C_{o j}\right)^{t}+\tau_{j} \Omega_{x_{2 j}}^{t} 2_{x j} \Omega_{d_{2 j}},
\end{gathered}
$$

where

$$
\begin{gathered}
L_{j}=\Omega_{d_{2 j}}^{t} G_{c j}^{t} B_{o j}^{t} \Omega_{d_{1 j}}^{-1}\left(C_{o j}+E_{o j}\right)^{\dagger} E_{o j}, \\
\Pi_{d 1 j}=A_{o d j} \Omega_{d_{1 j}}+\Omega_{d_{1 j}}^{t} A_{o d j}^{t}+\Gamma_{j} H_{j}^{-1} \Gamma_{j}^{t}+\mathscr{R}_{\sigma 1 j}, \\
\Pi_{d 2 j}=\left(A_{o j}+B_{o j} D_{o j}^{\dagger} C_{o j}\right) \Omega_{d_{2 j}}+\Omega_{d_{2 j}}^{t}\left(A_{o j}+B_{o j} D_{o j}^{\dagger} C_{o j}\right)^{t}+\mathscr{R}_{\sigma 2 j},
\end{gathered}
$$

and the feedback gains are given by

$$
G_{c j}=D_{o j}^{\dagger} C_{o j}, \quad K_{c j}=-\Omega_{d_{2 j}}^{t} G_{c j}^{t} B_{o j}^{t} \Omega_{d_{1 j}}^{-1}\left(C_{o j}+E_{o j}\right)^{\dagger}
$$

Proof. Using the block-diagonal matrices

$$
\Omega_{\sigma j}=\left[\begin{array}{cc}
\Omega_{\sigma 1 j} & 0 \\
0 & \Omega_{\sigma 2 j}
\end{array}\right], \quad \Omega_{x j}=\left[\begin{array}{cc}
\Omega_{x 1 j} & 0 \\
0 & \Omega_{x 2 j}
\end{array}\right], \quad \Omega_{d j}=\left[\begin{array}{cc}
\Omega_{d 1 j} & 0 \\
0 & \Omega_{d 2 j}
\end{array}\right],
$$

then straightforward expansion of (4.12) with some matrix manipulations, we get equations and LMIs (4.14)-(4.15) such that inequality (4.12) is satisfied.

Remark 4.2. It is readily seen that the developed results for delay-dependent passivity (Theorem 2.3), state-feedback passivation (Theorem 3.1), and output-feedback passivation (Theorem 4.1) give less conservative results than those of [9, 15-19, 21-25] since they provide a new state transformation for delay-dependent passive analysis and synthesis. This represents our first major contribution in this paper. On the other hand, our control approach is technically superior to the techniques of $[24,29]$ since it establishes delay-dependent dynamic controllers for interconnected time-delay systems. 


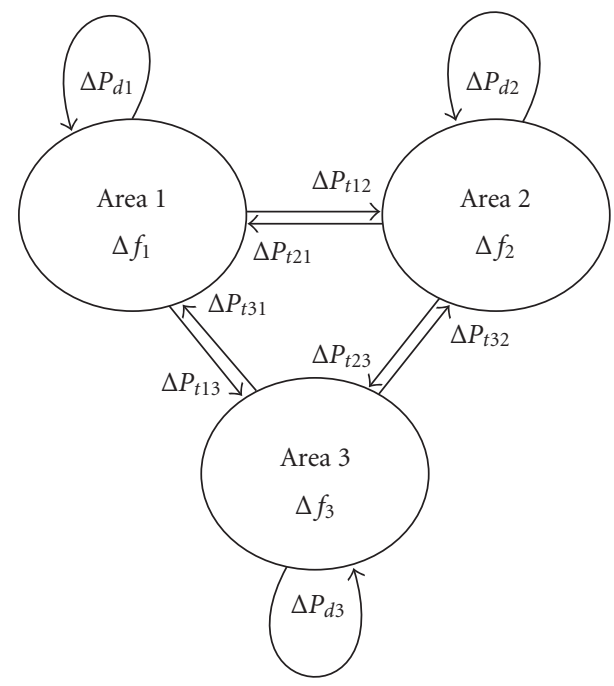

Figure 5.1. A schematic of three-area reheat power systems.

\section{Application to power systems}

In this section we demonstrate the application of the foregoing analytical developments to multiarea reheat power systems, a schematic layout of which in case of three-area is shown in Figure 5.1.

We will show that the resulting linearized dynamical model can be cast into the format (2.6). In modeling the power system, we note that the overall system model would essentially be an aggregate of the single-area reheat models in addition to the power interchange across the tie lines. By considering the dynamics of individual block of a singlearea reheat power system as well as the integral area control [6], the corresponding governing relations are derived below. First, the dynamic model of the generator is described by [29]

$$
\begin{gathered}
\dot{\delta}(t)=\omega_{o} \Delta \omega(t), \\
\Delta \dot{\omega}(t)=\frac{1}{M}\left[P_{M}+r_{g}(t)+K_{d} \Delta \omega(t)-P_{e}\right], \\
\dot{E}_{q}^{\prime}(t)=\frac{1}{T_{d o}^{\prime}}\left[E_{f d}(t)-\left[x_{d}-x_{d}^{\prime}\right] i_{d}-E_{q}^{\prime}(t)\right],
\end{gathered}
$$

where

$$
i_{d}=\frac{E_{q}-E \cos \delta}{x_{e}+x_{q}}, \quad E_{q}=E_{q}^{\prime}+\left[x_{d}-x_{d}^{\prime}\right] i_{d}, \quad P_{e}=\frac{E_{q}^{\prime} E \sin \delta}{\left[x_{d}-x_{d}^{\prime}\right]},
$$


which leads to

$$
i_{d}=\frac{E_{q}-E \cos \delta}{x_{e}+x_{q}-x_{d}+x_{q}^{\prime}} .
$$

Then the automatic excitation voltage regulator is described by

$$
\dot{E}_{f d}(t)=\frac{K_{A}}{T_{A}}\left[V_{r}-V_{t}+V_{\mathrm{pss}}(t)\right]-\frac{K_{f d}(t)}{T_{A}} .
$$

The governor and the power system stabilizer are represented by

$$
\begin{gathered}
\dot{g}=\frac{1}{T_{g}}[b \Delta \omega(t-\tau)-g(t)], \quad r_{g}=a \Delta \omega(t-\tau)+b g(t), \\
\dot{y}_{1}(t)=y_{2}(t), \quad \dot{y}_{2}(t)=\frac{-1}{T_{2} T_{Q}}\left[y_{1}(t)+\left(T_{2}+T_{Q}\right) y_{2}(t)-\Delta \omega(t-\tau)\right], \\
V_{\mathrm{pss}}=\frac{-K_{J}}{K_{A}}\left[\frac{-T_{1}}{T_{2}} y_{1}(t)+\frac{T_{Q}-T_{1} T_{2}-T_{1} T_{Q}}{T_{2}} y_{2}(t)+\frac{T_{1}}{T_{2}} \Delta \omega(t-\tau)\right] .
\end{gathered}
$$

By linearizing the dynamical relationships (5.1)-(5.5) around an operating point $\left(\delta_{o}, \omega_{o}\right.$, $\left.E_{q o}^{\prime}, E_{f d o}, g_{o}, y_{1 o}, y_{2 o}\right)$ and defining the deviations

$$
\begin{gathered}
\Delta \delta=\delta-\delta_{o}, \quad \Delta \omega=\omega-\omega_{o}, \quad \Delta E_{q}^{\prime}=E_{q}^{\prime}-E_{q o}^{\prime}, \quad \Delta E_{f d}=E_{f d}-E_{f g o}, \\
\Delta g=g-g_{o}, \quad \Delta y_{1}=y_{1}-y_{1 o}, \quad \Delta y_{2}=y_{2}-y_{2 o},
\end{gathered}
$$

we introduce the state and input vectors as $x^{t}=\left[\Delta \delta, \Delta \omega, \Delta E_{q}^{\prime}, \Delta E_{f d}, \Delta g, \Delta y_{1}, \Delta y_{2}\right]$ and $u=\left[\Delta P_{M},\left(V_{\text {ref }}-V_{t}\right)\right]$. Then the resulting linearized model can be cast into the form (2.6)-(2.7) with

$$
A_{o}=\left[\begin{array}{ccccccc}
0 & \omega_{o} & 0 & 0 & 0 & 0 & 0 \\
G_{1} & \frac{K_{d}}{M} & G_{2} & 0 & \frac{b}{M} & 0 & 0 \\
G_{3} & 0 & G_{4} & \frac{1}{T_{d o}^{\prime}} & 0 & 0 & 0 \\
0 & 0 & 0 & -\frac{1}{T_{A}} & 0 & G_{5} & G_{6} \\
0 & 0 & 0 & 0 & -\frac{1}{T_{g}} & 0 & 0 \\
0 & 0 & 0 & 0 & 0 & 0 & 1 \\
0 & 0 & 0 & 0 & 0 & G_{7} & G_{8}
\end{array}\right],
$$




$$
\begin{aligned}
& A_{d}=\left[\begin{array}{ccccccc}
0 & 0 & 0 & 0 & 0 & 0 & 0 \\
0 & \frac{a}{M} & 0 & 0 & 0 & 0 & 0 \\
0 & 0 & 0 & 0 & 0 & 0 & 0 \\
0 & K_{9} & 0 & 0 & 0 & 0 & 0 \\
0 & K_{10} & 0 & 0 & 0 & 0 & 0 \\
0 & 0 & 0 & 0 & 0 & 0 & 0 \\
0 & K_{11} & 0 & 0 & 0 & 0 & 0
\end{array}\right] \\
& B_{0}=\left[\begin{array}{cc}
0 & 0 \\
\frac{1}{M} & 0 \\
0 & 0 \\
0 & \frac{K_{A}}{T_{A}} \\
0 & 0 \\
0 & 0 \\
0 & 0
\end{array}\right], \quad C_{o}^{t}=\left[\begin{array}{cc}
1 & 0 \\
0 & 1 \\
0 & 0 \\
0 & 0 \\
0 & 0 \\
0 & 0 \\
0 & 0
\end{array}\right], \quad C_{d}^{t}=\left[\begin{array}{ll}
0 & 0 \\
0 & 1 \\
0 & 0 \\
0 & 0 \\
0 & 0 \\
0 & 0 \\
0 & 0
\end{array}\right],
\end{aligned}
$$

where

$$
\begin{gathered}
G_{1}=-\frac{E_{q o}^{\prime} E \cos \delta_{o}}{M\left[x_{e}+x_{d}^{\prime}\right]}, \quad G_{2}=-\frac{E_{q o}^{\prime} E \sin \delta_{o}}{M\left[x_{e}+x_{d}^{\prime}\right]}, \quad G_{3}=-E \sin \delta_{o} \frac{x_{d}-x_{d}^{\prime}}{T_{d o}^{\prime}\left[x_{e}+x_{q}-x_{d}+x_{d}^{\prime}\right]}, \\
G_{4}=-\frac{x_{e}+x_{q}}{T_{d o}^{\prime}\left[x_{e}+x_{q}-x_{d}+x_{d}^{\prime}\right]}, \quad G_{5}=\frac{K_{J} T_{1}}{T_{A} T_{2}}, \quad G_{6}=-\frac{K_{J}\left[T_{Q}-T_{1} T_{2}-T_{1} T_{Q}\right]}{T_{A} T_{2}}, \\
G_{7}=-\frac{1}{T_{2} T_{Q}}, \quad G_{8}=\frac{T_{2}+T_{Q}}{T_{2} T_{Q}}, \quad G_{9}=-\frac{K_{J} T_{1}}{T_{A} T_{2}}, \quad G_{10}=\frac{b}{T_{g}}, \quad G_{11}=\frac{1}{T_{2} T_{Q}} .
\end{gathered}
$$

It should be observed that the delay factor $\tau$ occurs within the dynamics of the governor and the power system stabilizer and the turbine reheat power. This is typical in large power systems and an upper bound on the delay factor can be estimated using offline simulation. This confirms our observation that the linearized dynamical model of multiarea reheat power systems fits nicely the frame of interconnected time-delay systems.

5.1. Numerical data. Assuming identical power areas, the numerical values of the different parameters of any of the areas are given by

(i) synchronous machine parameters

$$
\begin{gathered}
\omega_{o}=314.16 \mathrm{rad} / \mathrm{s}, \quad M=6.92, \quad K_{d}=-0.027, \quad x_{d}=1.24 \mathrm{pu}, \\
x_{q}=0.743 \mathrm{pu}, \quad x_{d}^{\prime}=0.022 \mathrm{pu}, \quad x=0.8 \mathrm{pu}, \quad P_{M}=0.437 \mathrm{pu}, \\
E=1 \mathrm{pu}, \quad T_{d o}^{\prime}=5 \mathrm{~s}, \quad \delta_{o}=20^{\circ}, \quad E_{q o}^{\prime}=1.05 ;
\end{gathered}
$$


(ii) voltage regulator and excitation system

$$
K_{A}=250, \quad T_{A}=0.001 \mathrm{~s}, \quad V_{\text {ref }}=1 \mathrm{pu} ;
$$

(iii) governor system parameters

$$
a=-0.000124, \quad b=-0.17, \quad T_{g}=0.25 \mathrm{~s} ;
$$

(iv) power system stabilizer

$$
K_{J}=12, \quad T_{Q}=2.5 \mathrm{~s}, \quad T_{1}=0.1 \mathrm{~s}, \quad T_{2}=0.03 \mathrm{~s} .
$$

The upper bound on the time-delay $\tau^{*}$ is 0.65 . Application of Theorem 4.1 yields the gains of the dynamic output feedback controller:

$$
K_{c j}=\left[\begin{array}{cc}
0 & -0.03 \\
-0.02 & 1.25 \\
0 & -0.02 \\
0 & -0.001 \\
0 & -0.006 \\
0 & -0.07 \\
0.004 & -0.52
\end{array}\right], \quad G_{c j}=\left[\begin{array}{ccccccc}
-0.04 & -0.0006 & 0.4 & 0.02 & 0.19 & 0 & 0 \\
0 & -0.0001 & 0 & 0.004 & 0-0.16 & 3.6 &
\end{array}\right] .
$$

5.2. Simulation results. Extensive simulations were carried out to examine the capabilities of the developed passivity-based control design and to compare the ensuing results with the $\mathscr{H}_{\infty}$-controller developed in [29]. The three-area interconnected power system was subjected to a constant load disturbance of $10 \%$ of the rated capacity. For purpose of comparison with other related works and due to symmetry, we consider the case when the disturbance is affecting one area only (say, area 1). Here the tie-line power interchange error between the other two areas (area 2 and area 3 in this case) is zero. It should be noted that all tie-line power interchange errors between the different areas with and without integral control are zero. This means that the tie lines between the different areas cannot carry any extra interchanging power due to the load increase in the areas.

The simulation results of the areas are displayed in Figures 5.2-5.7. In Figures 5.2 and 5.3 , the frequency error and the inter-area power exchange error in area 1 are plotted for the cases with and without integral control using the developed passivity method. The corresponding results for areas 2 and 3 are plotted in Figures 5.4 and 5.5. Finally, in Figures 5.6 and 5.7, the frequency error and the inter-area power exchange error are plotted using three methods: standard linear-quadratic, the $\mathscr{H}_{\infty}$-controller of [29], and the passivity method developed in this paper. From these results, we observe that the steady-state frequency errors in each area were eliminated in a relatively short time. The inter-area tie-line power exchange errors are zero due to the fact that each area is capable of absorbing its own load increase.

In comparison to other established control design methods, the developed passivitybased control design for the interconnected power system has been proven to attain a satisfactory performance. 


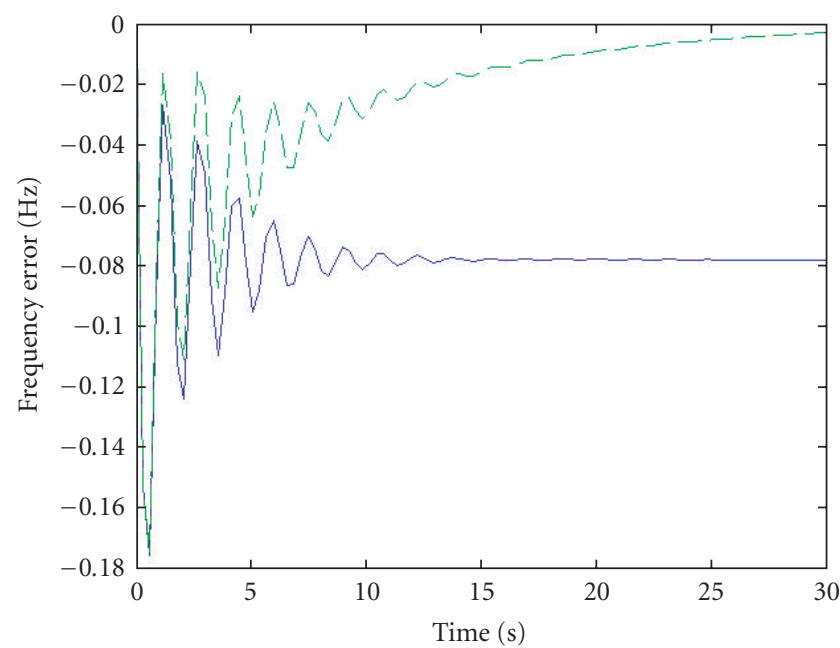

With integral control

- - Without integral control

Figure 5.2. Frequency error in area 1: with integral control (solid line); without integral control (dashed line).

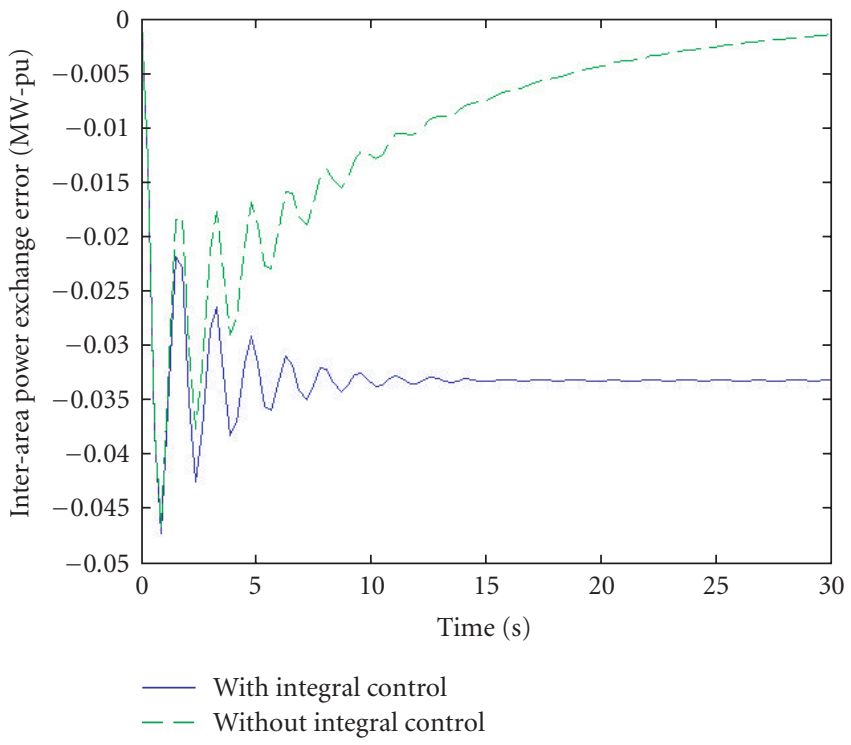

Figure 5.3. Inter-area power exchange error in area 1: with integral control (solid line); without integral control (dashed line). 


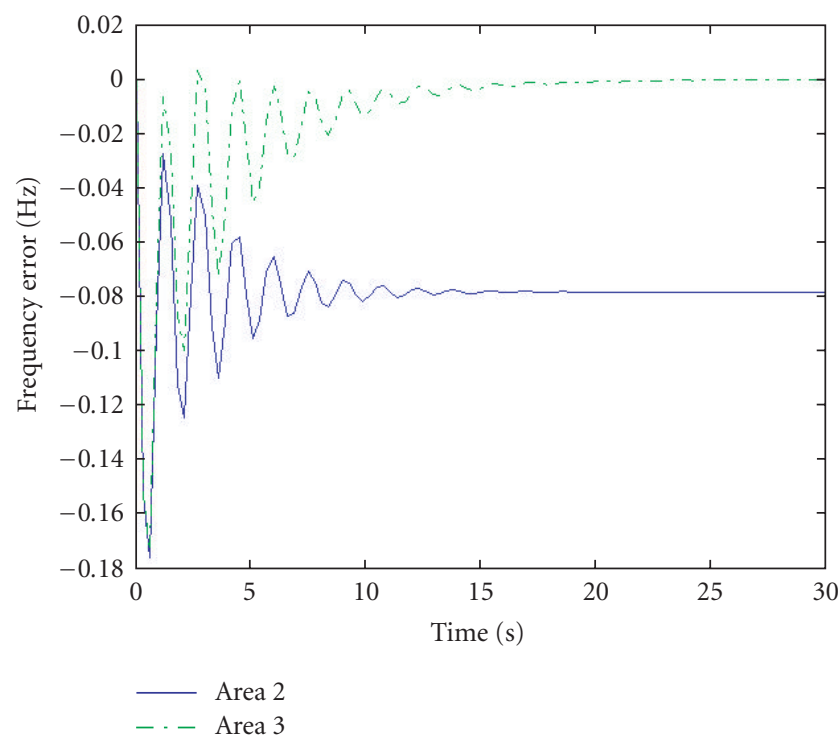

Figure 5.4. Frequency errors: in area 2 (solid line); in area 3 (dashed line).

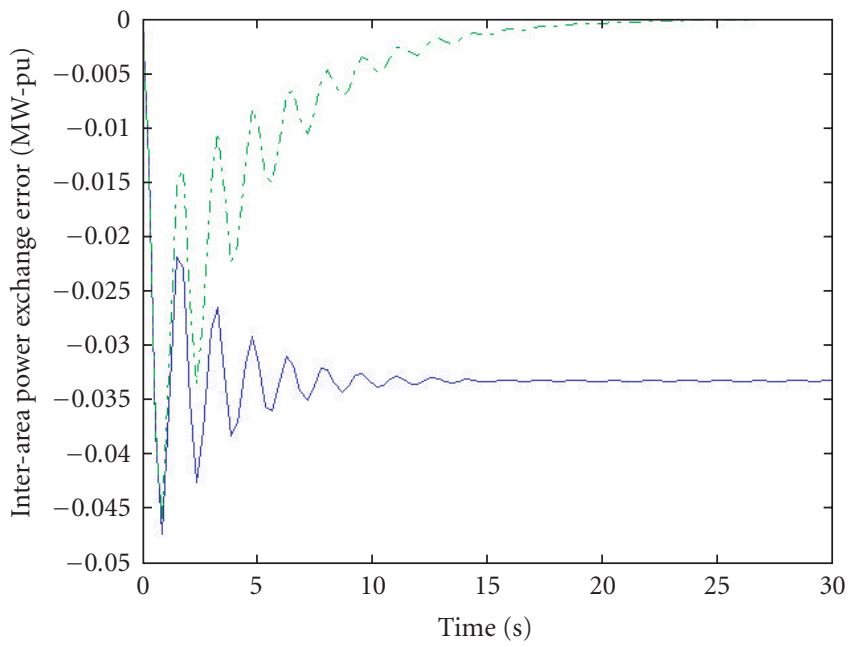

Area 2

Area 3

Figure 5.5. Inter-area power exchange errors: in area 2 (solid line); in area 3 (dashed line). 


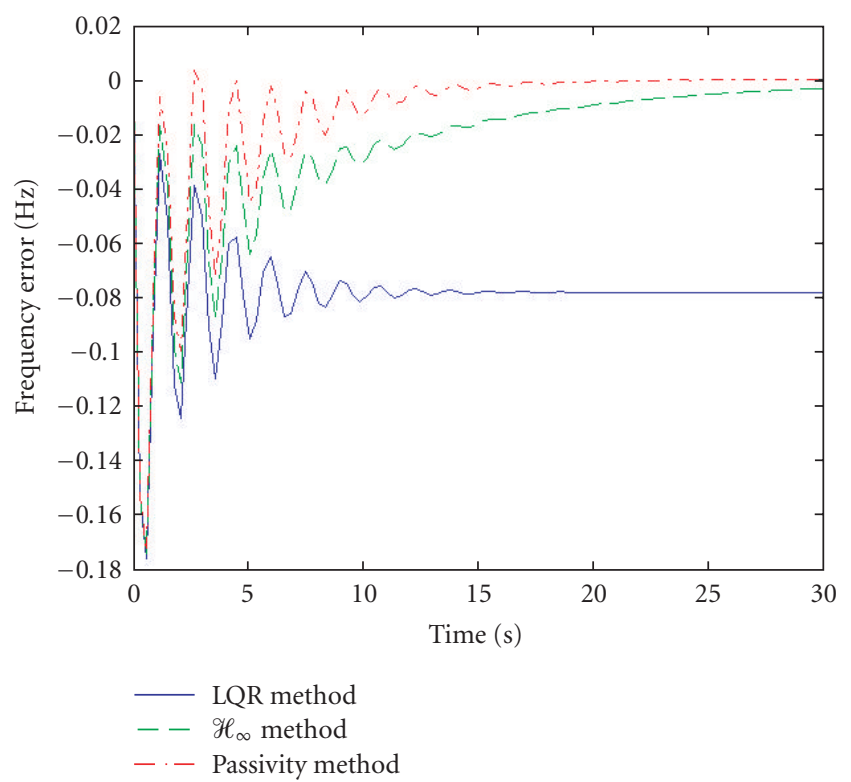

Figure 5.6. Frequency error in area 1: LQR method (solid line); $\mathscr{H}_{\infty}$ method [29] (dashed line); passivity method (dashed-dotted line).

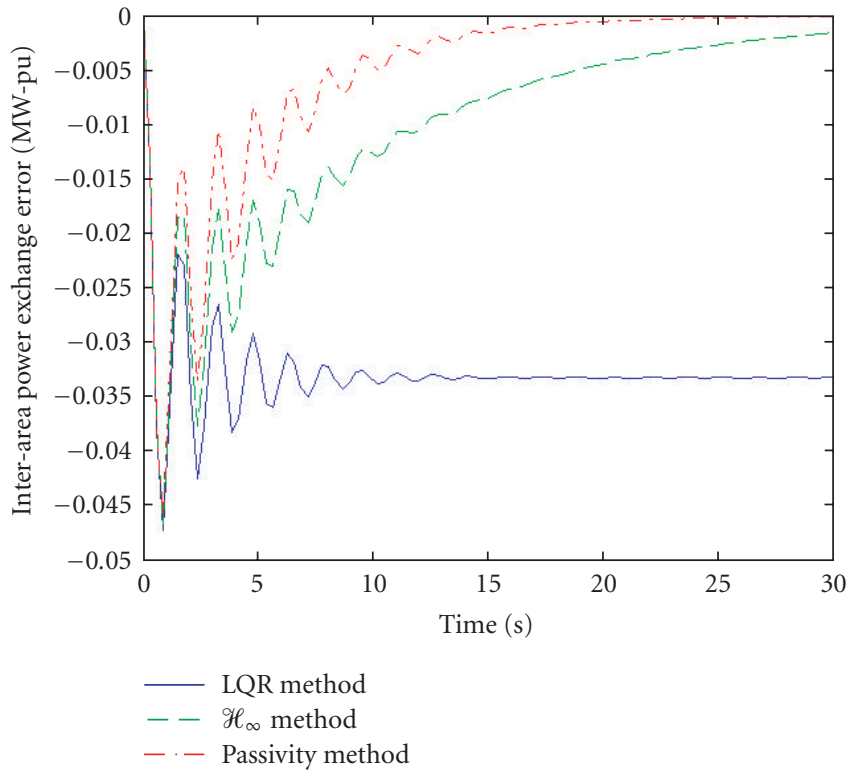

Figure 5.7. Inter-area power exchange error in area 1: LQR method (solid line); $\mathscr{H}_{\infty}$ method [29] (dashed line); passivity method (dashed-dotted line). 


\section{Conclusions}

A new state transformation has been developed to exhibit the delay-dependence dynamics of a class of linear interconnected time-delay systems for the purpose of investigating the problems of passivity and passivation. It has been established that the passivity condition can be cast in a linear matrix inequality (LMI) format at the subsystem level. For state-feedback passivation, it is proven that it is indifferent to use instantaneous or delayed decentralized state feedback. The case of output-feedback passivation is also treated using local dynamic controllers. Application to multiarea reheat power systems has been given to support the analytical results.

\section{Acknowledgment}

This work was supported in part by UAE Research Affairs under Grant no. 03-04-7-11/03.

\section{References}

[1] N. F. Al-Muthairi, M. Zribi, and M. S. Mahmoud, Adaptive identification and control for a class of interconnected systems, Systems Analysis Modelling Simulation 42 (2002), no. 6, 851-877.

[2] B. O. D. Anderson and S. Vongpanitherd, Modern Analysis and Synthesis: A Modern Systems Theory Approach, Prentice-Hall, New Jersey, 1973.

[3] A. A. Bahnasawi, A. S. Al-Fuhaid, and M. S. Mahmoud, A new hierarchical control structure for a class of uncertain discrete systems, Control Theory and Advanced Technology 6 (1990), no. 1, $1-21$.

[4] _ Decentralized and hierarchical control of interconnected uncertain systems, IEE Proceedings. Part D. Control Theory and Applications 137 (1990), 311-321.

[5] A. A. Bahnasawi and M. S. Mahmoud, Control of Partially-Known Dynamical Systems, Lecture Notes in Control and Information Sciences, vol. 124, Springer, Berlin, 1989.

[6] A. R. Bergen and V. Vittal, Power Systems Analysis, Prentice-Hall, New Jersey, 2000.

[7] S. Boyd, L. El Ghaoui, E. Feron, and V. Balakrishnan, Linear Matrix Inequalities in System and Control Theory, SIAM Studies in Applied Mathematics, vol. 15, SIAM, Pennsylvania, 1994.

[8] G. Escobar, A. J. van der Schaft, and R. Ortega, A Hamiltonian viewpoint in the modeling of switching power converters, Automatica 35 (1999), no. 3, 445-452.

[9] E. Fridman and U. Shaked, On delay-dependent passivity, IEEE Transactions on Automatic Control 47 (2002), no. 4, 664-669.

[10] P. Gahinet, A. Nemirovski, A. L. Laub, and M. Chilali, LMI Control Toolbox, The MathWorks, Massachusetts, 1995.

[11] J. C. Geromel, J. Bernussou, and P. L. D. Peres, Decentralized control through parameter space optimization, Automatica 30 (1994), no. 10, 1565-1578.

[12] Z. Hu, Decentralized stabilization of large scale interconnected systems with delays, IEEE Transactions on Automatic Control 39 (1994), no. 1, 180-182.

[13] T. N. Lee and U. L. Radovic, Decentralized stabilization of linear continuous and discrete-time systems with delays in interconnections, IEEE Transactions on Automatic Control 33 (1988), no. 8, 757-761.

[14] R. Lozano, B. Brogliato, O. Egeland, and B. Maschke, Dissipative Systems Analysis and Control. Theory and Applications, Communications and Control Engineering Series, Springer, London, 2000.

[15] M. S. Mahmoud, Stabilizing control for a class of uncertain interconnected systems, IEEE Transactions on Automatic Control 39 (1994), no. 12, 2484-2488. 
[16] _ Guaranteed stabilization of interconnected discrete-time uncertain systems, International Journal of Systems Science 26 (1995), no. 2, 337-358.

[17] _ Adaptive stabilization of a class of interconnected systems, Computers \& Electrical Engineering 23 (1997), no. 4, 225-238.

[18]__ Passive control synthesis for uncertain time-delay systems, Proceedings of the 37th IEEE Conference on Decision and Control, Florida, 1998, pp. 4139-4143.

[19] _ Robust Control and Filtering for Time-Delay Systems, Control Engineering (New York), vol. 5, Marcel Dekker, New York, 2000.

[20] M. S. Mahmoud and N. F. Al-Muthairi, Design of robust controllers for time-delay systems, IEEE Transactions on Automatic Control 39 (1994), no. 5, 995-999.

[21] M. S. Mahmoud and S. Bingulac, Robust design of stabilizing controllers for interconnected timedelay systems, Automatica 34 (1998), no. 6, 795-800.

[22] M. S. Mahmoud and L. Xie, Stability and positive realness of time-delay systems, Journal of Mathematical Analysis and Applications 239 (1999), no. 1, 7-19.

[23] _ Passivity analysis and synthesis for uncertain time-delay systems, Mathematical Problems in Engineering 7 (2001), no. 5, 455-484.

[24] M. S. Mahmoud and M. Zribi, Robust and $\mathscr{H}_{\infty}$ stabilization of interconnected systems with delays, IEE Proceedings. Part D. Control Theory and Applications 145 (1998), no. 6, 559-567.

[25] _ Passive control synthesis for uncertain systems with multiple-state delays, Computers \& Electrical Engineering 28 (2002), no. 3, 195-216, (special issue on Time-Delay Systems).

[26] C. J. Mao and J.-H. Yang, Decentralized output tracking for linear uncertain interconnected systems, Automatica 31 (1995), no. 1, 151-154.

[27] W. Sun, P. P. Khargonekar, and D. Shim, Solution to the positive real control problem for linear time-invariant systems, IEEE Transactions on Automatic Control 39 (1994), no. 10, 2034-2046.

[28] Z. Xi, G. Feng, D. Cheng, and Q. Lu, Nonlinear decentralized saturated controller design for power systems, IEEE Transactions on Control Systems Technology 11 (2003), no. 4, 539-547.

[29] M. Zribi, M. S. Mahmoud, M. Karkoub, and T. Li, $\mathscr{H}_{\infty}$ controllers for linearised time-delay power systems, IEE Proceedings - Generation, Transmission and Distribution 147 (2000), no. 6, 401408.

Magdi S. Mahmoud: C-Group Consultants, P.O. Box 293 Orman, Dokki, 12311 Giza, Egypt E-mail address: magdim@yahoo.com

Abdulla Ismail: College of Engineering, United Arab Emirates University, P.O. Box 17555,

Al-Ain, United Arab Emirates

E-mail address: abdulla@uaeu.ac.ae 


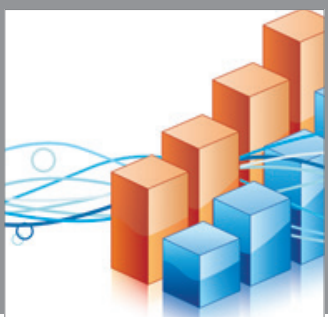

Advances in

Operations Research

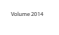

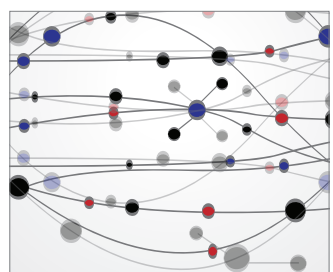

\section{The Scientific} World Journal
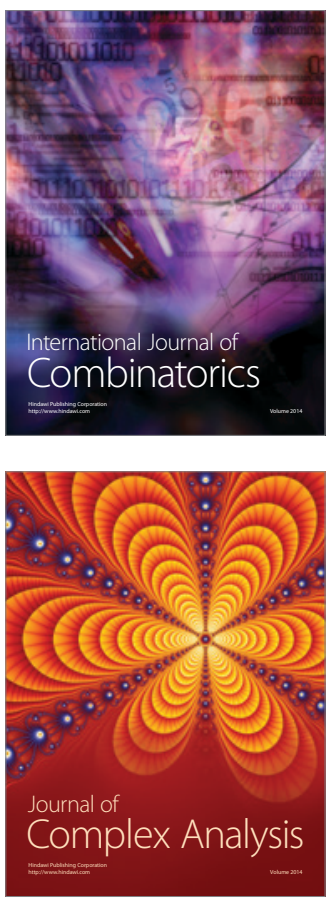

International Journal of

Mathematics and

Mathematical

Sciences
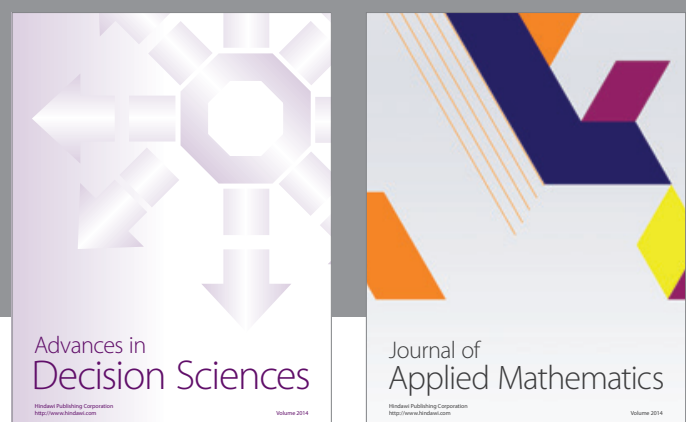

Journal of

Applied Mathematics
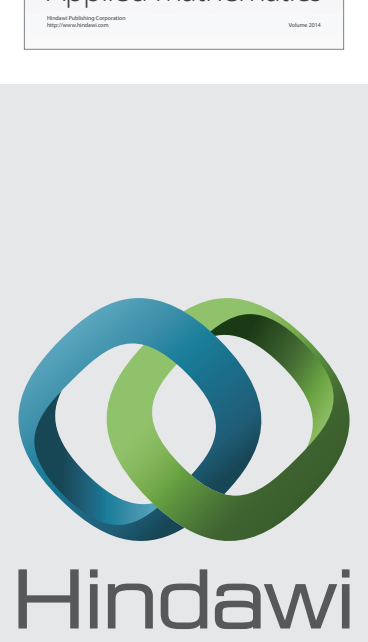

Submit your manuscripts at http://www.hindawi.com
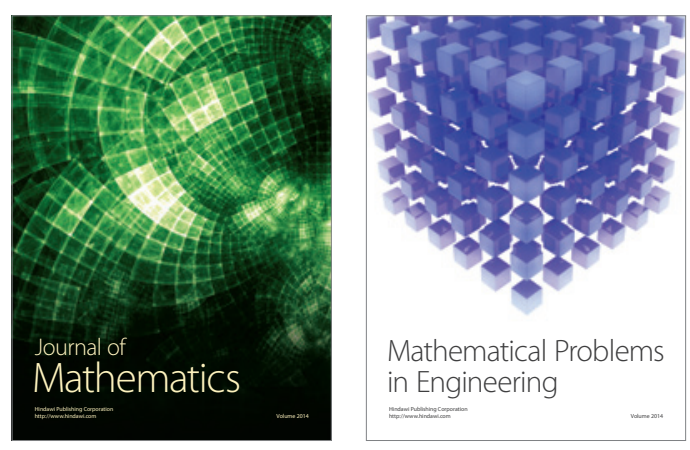

Mathematical Problems in Engineering
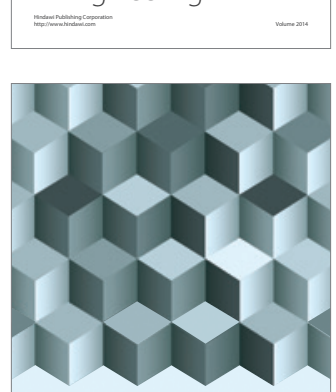

Journal of

Function Spaces
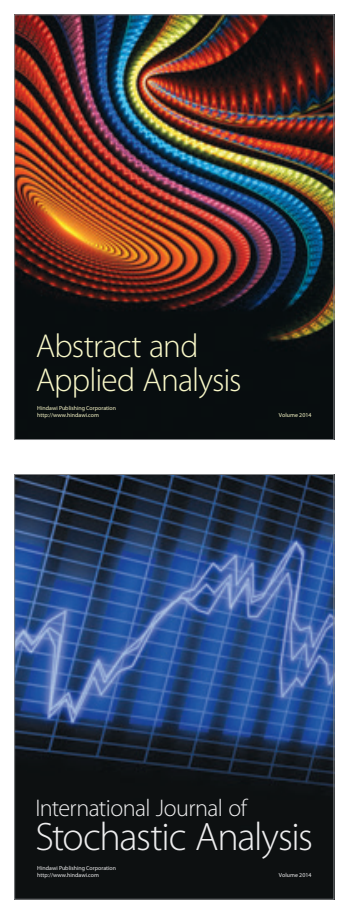

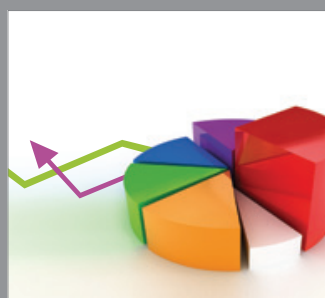

ournal of

Probability and Statistics

Promensencen
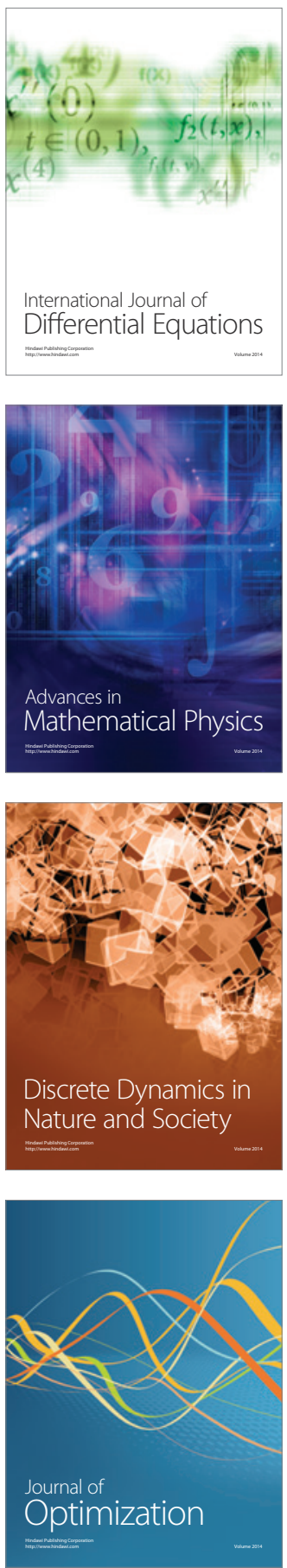